\section{Extracorporeal cardiopulmonary resuscitation among patients with out-of-hospital cardiac arrest}

\author{
Dae-Hee Choi', Youn-Jung Kim ${ }^{1}$, Seung Mok Ryoo', Chang Hwan Sohn', \\ Shin Ahn', Dong-Woo Seo', Ju Yong Lim², Won Young Kim \\ 'Department of Emergency Medicine, Asan Medical Center, University of Ulsan College of Medicine, Seoul, \\ Korea \\ ${ }^{2}$ Department of Thoracic and Cardiovascular Surgery, Asan Medical Center, University of Ulsan College of \\ Medicine, Seoul, Korea
}

Objective Extracorporeal cardiopulmonary resuscitation (ECPR) may be considered as a rescue therapy for patients with refractory cardiac arrest. Identifying patients who might benefit from this potential life-saving procedure is crucial for implementation of ECPR. The objective of this study was to estimate the number of patients who fulfilled a hypothetical set of ECPR criteria and to evaluate the outcome of ECPR candidates treated with conventional cardiopulmonary resuscitation.

Methods We performed an observational study using data from a prospective registry of consecutive adults ( $\geq 18$ years) with non-traumatic out-of-hospital cardiac arrest in a tertiary hospital between January 2011 and December 2015. We developed a hypothetical set of ECPR criteria including age $\leq 75$ years, witnessed cardiac arrest, no-flow time $\leq 5$ minutes, low-flow time $\leq 30$ minutes, refractory arrest at emergency department $>10$ minutes, and no exclusion criteria. The primary endpoint was the proportion of good neurologic outcome of ECPR-eligible patients.

Results Of 568 out-of-hospital cardiac arrest cases, 60 cases (10.6\%) fulfilled our ECPR criteria. ECPR was performed for 10 of 60 ECPR-eligible patients (16.7\%). Three of the 10 patients with ECPR (30.0\%), but only 2 of the other 50 patients without ECPR (4.0\%) had a good neurologic outcome at 1 month.

Conclusion ECPR implementation might be a rescue option for increasing the probability of survival in potentially hopeless but ECPR-eligible patients.

Keywords Out-of-hospital cardiac arrest; Cardiopulmonary resuscitation; Extracorporeal membrane oxygenation

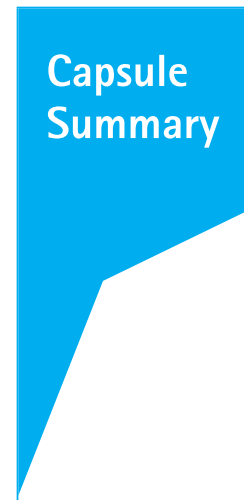

What is already known

Extracorporeal cardiopulmonary resuscitation (ECPR) for cardiac arrest may be considered as a rescue therapy for refractory cardiac arrest patients. Selection of patients for ECPR is especially important for successful implementation of ECPR strategy for out-of-hospital cardiac arrest patients.

What is new in the current study

We found $10 \%$ of out-of-hospital cardiac arrest patients were potential candidates for ECPR and that 30\% of patients with ECPR had a good neurological outcome, whereas only $4 \%$ of those with conventional cardiopulmonary resuscitation did.
eISSN: $2383-4625$

Received: 6 May 2016

Revised: 31 May 2016

Accepted: 14 June 2016

Correspondence to: Won Young Kim Department of Emergency Medicine, Asan Medical Center, University of Ulsan College of Medicine, 88 Olympicro 43-gil, Songpa-gu, Seoul 05505, Korea

E-mail:wonpia73@naver.com

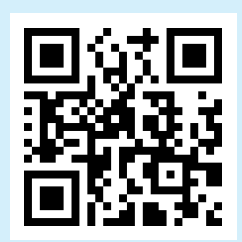

How to cite this article:

Choi DH, Kim YJ, Ryoo SM, Sohn CH, Ahn S, Seo DW, Lim JY, Kim WY. Extracorporeal cardiopulmonary resuscitation among patients with out-of-hospital cardiac arrest. Clin Exp Emerg Med 2016;3(3):132-138.

This is an Open Access article distributed under the terms of the Creative Commons Attribution Non-Commercial License (http:// creativecommons.org/licenses/by-nc/4.0/). 


\section{INTRODUCTION}

Despite recent improvements in advanced life support, the reported overall rate of survival to hospital discharge is $10.6 \%$ and the rate of survival with good neurologic function is 8.3\%.' Extracorporeal cardiopulmonary resuscitation (ECPR) is the use of venoarterial extracorporeal membrane oxygenation (ECMO) to provide support after conventional cardiopulmonary resuscitation (CPR) has failed to restore circulation. Studies have shown ECPR to improve the survival rate among refractory cardiac arrest patients. ${ }^{2-8}$ Although there is insufficient evidence to recommend the routine use of ECPR, ECPR may have a role as a rescue therapy in selected patients in whom the suspected etiology of cardiac arrest is potentially reversible.

The implementation of ECPR requires a specially trained team that may include physicians, surgeons, perfusionists, and skilled nursing staff as well as specialized protocols, equipment, and hospital resources. ${ }^{2,4}$ Therefore, selection of suitable patients is one of the important factors for success. ${ }^{8}$ Observational studies have shown that ECPR for cardiac arrest is associated with improved survival when the cause of cardiac arrest is reversible (e.g., myocardial infarction, pulmonary embolism, severe hypothermia, and poisoning); the comorbidity is low; it is a case of witnessed cardiac arrest; the individual receives immediate high-quality CPR; and ECPR is implemented early (e.g., within 1 hour of collapse)., ${ }^{3,79-14}$ However, there are several uncertainties in the ECPR eligibility criteria. Moreover, the number of out-of-hospital cardiac arrest (OHCA) patients who can be candidates for ECPR has not been reported in South Korea.

The aim of this study was to estimate the number of patients who fulfilled a hypothetical set of ECPR criteria and to evaluate the outcome of ECPR candidates treated with conventional CPR.

\section{METHODS}

\section{Study design and population}

This retrospective cohort study used data from a prospective registry and was conducted at the emergency department (ED) of a university-affiliated teaching hospital in Seoul, Korea, with an annual census of approximately 100,000 visits, between January 1, 2011, and December 31, 2015. Before commencing the study, the institutional review board of the hospital approved our study and waived the requirement for informed consent because of its retrospective design.

In this study, all consecutive adults (age $\geq 18$ years) with nontraumatic OHCA were included, and their electronic medical records were reviewed thoroughly by investigators. Emergency med- ical service (EMS) providers in Korea are instructed to scoop and run to the ED while performing CPR during ambulance transport as soon as possible after performing 1 cycle of CPR. In the field, EMS personnel are not legally allowed to declare death.

Despite the lack of a standardized ECPR strategy and heterogeneity in previous ECPR studies, some factors have been commonly suggested as the inclusion and exclusion criteria for receiving ECPR. Based on previous studies, we developed a hypothetical set of ECPR eligibility criteria and applied the criteria to our cohort to determine the number of OHCA patients who could be candidates for ECPR. The ECPR eligibility criteria were age $\leq 75$ years, witnessed cardiac arrest, bystander administration of CPR or no-flow time $\leq 5$ minutes, prehospital low-flow time $\leq 30 \mathrm{~min}$ utes and refractory arrest $>10$ minutes of conventional CPR at the ED, known absence of severe comorbidities that preclude admission to the intensive care unit, and absence of all exclusion criteria. The exclusion criteria were do-not-resuscitate order, a poor performance status or terminal illness that preceded the arrest due to malignancy or neurologic disease, trauma, intracranial hemorrhage, acute aortic dissection with pericardial effusion observed by echocardiography, and achievement of sustained return of spontaneous circulation within 10 minutes after ED arrival.

\section{Data collection}

Demographic data were obtained from EMS reports and medical records. We extracted the following data: demographic characteristics, cause of cardiac arrest, initial documented electrocardiogram rhythm at the scene, bystander administration of CPR, prehospital no-flow time, prehospital resuscitation time, and outcome including achievement of sustained return of spontaneous circulation, survival at 1 month after cardiac arrest, and good neurologic state defined as Cerebral Performance Category 1 or 2 at 1 month after cardiac arrest. Basic life support, advanced cardiovascular life support, and post-resuscitation care were performed in accordance with the current Advanced Cardiac Life Support Guidelines of 2010.

\section{Statistical analysis}

Continuous variables are expressed as mean \pm standard deviation when normally distributed and median with interquartile range when non-normally distributed. Categorical data are presented as absolute numbers and percentages. Differences between means were analyzed by the Student's t-test or the Mann-Whitney Utest. Differences between categorical variables were analyzed by the $\chi^{2}$ test or the Fisher's exact test, as appropriate. A 2-sided Pvalue $<0.05$ was considered statistically significant. All statistical analyses were performed using PASW Statistics ver. 18.0 (SPSS Inc., Chicago, IL, USA). 


\section{RESULTS}

Between January 1, 2011, and December 31, 2015, 568 adults with non-traumatic OHCA arrived in our ED (Fig. 1). Among these patients, the majority was excluded owing to the following reasons: age $>75$ years $(n=160,28.2 \%)$, unwitnessed arrest $(n=167$, $29.4 \%)$, no-flow time $>5$ minutes $(n=65,11.4 \%)$, prehospital low-flow time $>30$ minutes $(n=37,6.5 \%)$, and other reasons $(n=79,13.9 \%)$. Finally, 60 patients $(10.6 \%)$ met our ECPR criteria. Of these 60 patients, ECPR was performed for 10 patients, and conventional CPR was performed for the other 50 patients at our ED.

The demographic and baseline characteristics of the OHCA patients and ECPR-eligible patients are summarized in Tables 1 and 2, respectively. The prehospital clinical factors including age, sex, administration of CPR by bystanders, initial arrest rhythm, and prehospital low-flow time were similar between the 2 groups. However, ECPR patients were more likely to achieve sustained return of spontaneous circulation $(90.0 \%$ vs. $30.0 \%, P=0.001)$ and demonstrate a good neurologic outcome at 1 month $(30.0 \%$ vs. $4.0 \%, P=0.03$ ).

In all ECPR patients with good neurologic outcomes, the cardiac arrest was witnessed and bystanders administered CPR (Table 3). The mean prehospital low-flow time was 16 minutes in patients with good neurologic outcomes. The mean time to implantation of the ECMO set-up from ED arrival was 49 minutes in patients with good neurologic outcomes, and all these patients were treated with therapeutic hypothermia at $33^{\circ} \mathrm{C}$ for 24 hours as well
Table 1. Demographic and clinical data of OHCA patients

\begin{tabular}{|c|c|}
\hline Characteristics & Total OHCA patients $(n=568)$ \\
\hline Age (yr) & $65.0(51.3-77.0)$ \\
\hline Male sex & $369(65.0)$ \\
\hline $\begin{array}{l}\text { Witnessed } \\
\text { By emergency medical service providers } \\
\text { By lay person }\end{array}$ & $\begin{array}{r}348(61.3) \\
60(10.6) \\
288(50.7)\end{array}$ \\
\hline Bystander CPR & $320(56.3)$ \\
\hline $\begin{array}{l}\text { Initial rhythm at scene } \\
\text { Shockable } \\
\text { Unknown non-shockable } \\
\text { Pulseless electrical activity } \\
\text { Asystole }\end{array}$ & $\begin{array}{r}76(13.4) \\
87(15.3) \\
76(13.4) \\
329(57.9)\end{array}$ \\
\hline Prehospital no-flow time (min) & $3.0(0.0-8.0)$ \\
\hline Prehospital low-flow time (min) & $19.0(14.0-25.0)$ \\
\hline $\begin{array}{l}\text { Etiology } \\
\text { Cardiogenic } \\
\text { Respiratory } \\
\text { Other medical condition } \\
\text { Asphyxia } \\
\text { Bleeding } \\
\text { Others }\end{array}$ & $\begin{array}{l}236(41.5) \\
113(19.9) \\
94(16.5) \\
39(6.8) \\
23(4.0) \\
63(11.1)\end{array}$ \\
\hline Resuscitation duration in ED & $19.0(8.0-30.0)$ \\
\hline Sustained ROSC & $258(45.4)$ \\
\hline Admission & $158(27.8)$ \\
\hline Survival at 1 month & $53(9.3)$ \\
\hline \multicolumn{2}{|l|}{ Neurologic outcome at 1 month } \\
\hline CPC 1 & $18(34.0)$ \\
\hline $\mathrm{CPC} 2$ & $5(9.4)$ \\
\hline $\mathrm{CPC} 3$ & $6(11.3)$ \\
\hline $\mathrm{CPC} 4$ & $24(45.3)$ \\
\hline
\end{tabular}

Values are expressed as median with interquartile range or number (\%). OHCA, out-of-hospital cardiac arrest; $C P R$, cardiopulmonary resuscitation; ED, emergency department; ROSC, return of spontaneous circulation; CPC, Cerebral Performance Category.

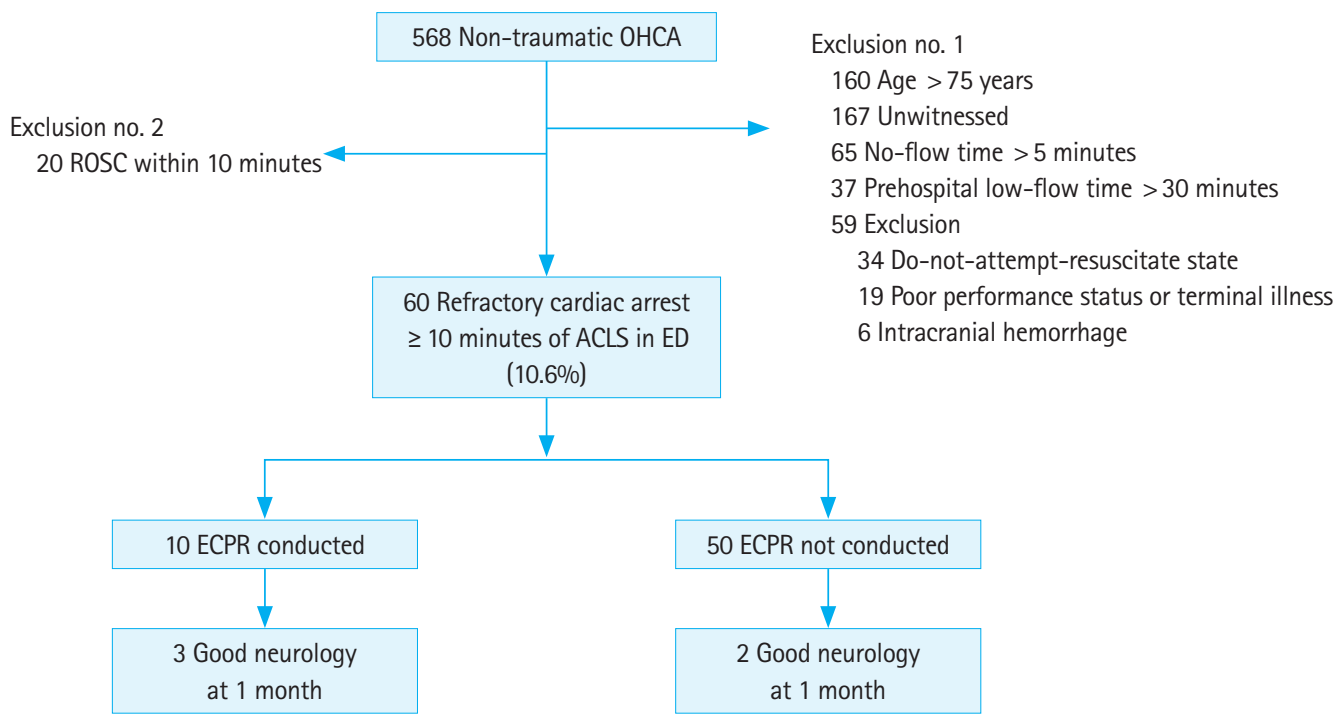

Fig. 1. Patient flow diagram. OHCA, out-of-hospital cardiac arrest; ROSC, return of spontaneous circulation; ACLS, advanced cardiovascular life support; $E D$, emergency department; ECPR, extracorporeal cardiopulmonary resuscitation. 
Table 2. Demographic and clinical data of patients who fulfilled the ECPR eligibility criteria

\begin{tabular}{|c|c|c|c|c|}
\hline & Total $(n=60)$ & ECPR patients $(n=10)$ & Non-ECPR patients $(n=50)$ & P-value \\
\hline Age (yr) & $58.9 \pm 11.2$ & $57.7 \pm 6.2$ & $59.2 \pm 12.0$ & 0.57 \\
\hline Male sex & $45(75.0)$ & $7(70.0)$ & $38(76.0)$ & 0.70 \\
\hline Witnessed & & & & 0.07 \\
\hline By EMS providers & $11(18.3)$ & $4(40.0)$ & $7(14.0)$ & \\
\hline By layperson & $49(81.7)$ & $6(60.0)$ & $43(86.0)$ & \\
\hline Bystander CPR & $49(81.7)$ & $8(80.0)$ & $41(82.0)$ & $>0.99$ \\
\hline Initial shockable rhythm at scene & $16(26.7)$ & $3(30.0)$ & $13(26.0)$ & $>0.99$ \\
\hline Prehospital low-flow time (min) & $18.0 \pm 8.1$ & $13.9 \pm 9.8$ & $18.8 \pm 7.6$ & 0.08 \\
\hline Presumed cardiogenic etiology & $38(63.3)$ & $9(90.0)$ & $29(58.0)$ & 0.08 \\
\hline Sustained ROSC & $24(40.0)$ & $9(90.0)$ & $15(30.0)$ & 0.001 \\
\hline Therapeutic hypothermia & $16(66.7)$ & $6(66.7)$ & $10(66.7)$ & $>0.99$ \\
\hline Percutaneous coronary intervention & $7(29.2)$ & $5(55.6)$ & $2(13.3)$ & 0.06 \\
\hline Survival at 1 month & $7(11.7)$ & $3(30.0)$ & $4(8.0)$ & 0.08 \\
\hline Good neurologic outcome at 1 month & $5(8.3)$ & $3(30.0)$ & $2(4.0)$ & 0.03 \\
\hline
\end{tabular}

Values are expressed as mean \pm standard deviation or number (\%).

ECPR, extracorporeal cardiopulmonary resuscitation; EMS, emergency medical service; CPR, cardiopulmonary resuscitation; ED, emergency department; ROSC, return of spontaneous circulation.

Table 3. Demographic and clinical data of ECPR patients who fulfilled our ECPR eligibility criteria

\begin{tabular}{|c|c|c|c|}
\hline & $\begin{array}{c}\text { Total } \\
(n=10)\end{array}$ & $\begin{array}{l}\text { Good neuro- } \\
\text { logic outcome } \\
\text { at } 1 \text { month } \\
(n=3)\end{array}$ & $\begin{array}{l}\text { Poor neuro- } \\
\text { logic outcome } \\
\text { at } 1 \text { month } \\
(n=7)\end{array}$ \\
\hline Age (yr) & $57.7 \pm 6.2$ & $55.7 \pm 5.7$ & $58.1 \pm 6.8$ \\
\hline Male sex & $7(70.0)$ & $3(100.0)$ & $4(57.1)$ \\
\hline \multicolumn{4}{|l|}{ Witnessed } \\
\hline By EMS providers & $4(40.0)$ & $2(66.7)$ & $2(28.6)$ \\
\hline By layperson & $6(60.0)$ & $1(33.3)$ & $5(71.4)$ \\
\hline Bystander CPR & $8(80.0)$ & $3(100.0)$ & $5(71.4)$ \\
\hline Initial shockable rhythm at scene & $3(30.0)$ & $2(66.7)$ & $1(14.3)$ \\
\hline Prehospital low-flow time (min) & $13.9 \pm 9.8$ & $16.0 \pm 11.5$ & $13.0 \pm 9.8$ \\
\hline Presumed cardiogenic etiology & $9(90.0)$ & $3(100.0)$ & $6(85.7)$ \\
\hline Time to ECPR from ED arrival & $50.5 \pm 22.0$ & $49.0 \pm 13.0$ & $57.9 \pm 22.2$ \\
\hline Therapeutic hypothermia & $6(60.0)$ & $3(100.0)$ & $3(42.9)$ \\
\hline Percutaneous coronary intervention & $5(50.0)$ & $3(100.0)$ & $2(28.6)$ \\
\hline Survival at 1 month & $3(30.0)$ & $3(100.0)$ & $0(0)$ \\
\hline \multicolumn{4}{|l|}{ Neurologic outcome at 1 month } \\
\hline CPC 1 & $2(66.7)$ & $2(66.7)$ & - \\
\hline $\mathrm{CPC} 2$ & $1(33.3)$ & $1(33.3)$ & - \\
\hline
\end{tabular}

Values are expressed as mean \pm standard deviation or number (\%).

ECPR, extracorporeal cardiopulmonary resuscitation; EMS, emergency medical service; CPR, cardiopulmonary resuscitation; ED, emergency department; $C P C$, Cerebral Performance Category.

as urgent percutaneous coronary intervention. The mean time to ECPR from ED arrival in patients with poor neurologic outcomes was prolonged to 58 minutes, and because of their hemodynamic instability, therapeutic hypothermia and percutaneous coronary intervention were performed selectively for $43 \%$ and $29 \%$ of the patients, respectively. All patients who had received ECPR but showed poor neurologic outcomes died within 1 month of ECPR.
Table 4. Demographic and clinical data of non-ECPR patients who fulfilled our ECPR eligibility criteria

\begin{tabular}{lrcc}
\hline & $\begin{array}{c}\text { Total } \\
(n=50)\end{array}$ & $\begin{array}{c}\text { Good neuro- } \\
\text { logic outcome } \\
\text { at } 1 \text { month } \\
(\mathrm{n}=2)\end{array}$ & $\begin{array}{c}\text { Poor neuro- } \\
\text { logic outcome } \\
\text { at } 1 \text { month } \\
(\mathrm{n}=48)\end{array}$ \\
\hline Age (yr) & $59.2 \pm 12.0$ & $64.0 \pm 9.9$ & $59.0 \pm 12.1$ \\
Male sex & $38(76.0)$ & $2(100.0)$ & $36(75.0)$ \\
Witnessed & $6(12.0)$ & $1(50.0)$ & $5(12.5)$ \\
$\quad$ By EMS providers & $43(86.0)$ & $1(50.0)$ & $42(87.5)$ \\
$\quad$ By layperson & $41(82.0)$ & $2(100.0)$ & $39(81.3)$ \\
Bystander CPR & $13(26.0)$ & $2(100.0)$ & $11(22.9)$ \\
Initial shockable rhythm at scene & $18.8 \pm 7.6$ & $22.0 \pm 4.2$ & $18.6 \pm 7.7$ \\
Prehospital low-flow time (min) & $29(58.0)$ & $2(100.0)$ & $27(56.3)$ \\
Presumed cardiogenic etiology & $32.1 \pm 14.0$ & $27.0 \pm 19.8$ & $32.3 \pm 14.0$ \\
ED resuscitation duration & $10(20.0)$ & $1(50.0)$ & $9(26.5)$ \\
Therapeutic hypothermia & $2(4.0)$ & $2(100.0)$ & $0(0)$ \\
Coronary angiography & $4(8.0)$ & $2(100.0)$ & $2(4.2)$ \\
Survival at 1 month & & &
\end{tabular}

Values are expressed as mean \pm standard deviation or number $(\%)$.

ECPR, extracorporeal cardiopulmonary resuscitation; EMS, emergency medical service; CPR, cardiopulmonary resuscitation; $E D$, emergency department.

All of the ECPR patients with poor neurologic outcomes died within 1 month of ECPR. The demographic and clinical factors of patients who received conventional CPR are shown in Table 4. Although the prehospital clinical factors were similar between ECPReligible patients who underwent ECPR and those who underwent conventional CPR, only 2 of 50 patients (4.0\%) had good neurologic outcome at 1 month without ECMO. Notably, witnessed arrest, administration of CPR by a bystander at that time, and initial shockable rhythm at the scene were reported in both the cases. 


\section{DISCUSSION}

In this study, we aimed to estimate the number of patients who could be candidates for ECPR therapy by using a hypothetical set of ECPR criteria and to determine the outcomes of ECPR-eligible patients treated with conventional resuscitation in order to estimate the potential benefits of ECPR. Sixty of 568 patients (10.6\%) fulfilled the hypothetical set of criteria for ECPR. Among these 60 patients, ECPR was performed for 10 patients, and 3 patients (30.0\%) had a good neurologic outcome at 1 month. Although the prehospital clinical factors were similar between ECPR and conventional CPR patients, only 2 of the 50 patients who underwent conventional CPR and fulfilled our ECPR criteria (4.0\%) demonstrated a good neurologic outcome at 1 month.

Several studies have reported favorable outcomes of ECPR for in-hospital cardiac arrest. ${ }^{3,15-18}$ However, several recent studies have reported conflicting experiences with ECPR in cases of OHCA., ${ }^{719,20}$ A recent meta-analysis reported that the rate of survival to discharge was lower in patients with OHCA who had received ECPR than that in patients with in-hospital cardiac arrest. ${ }^{21}$ However, the beneficial effect of ECPR compared with conventional CPR in OHCA patients was not clear for survival to discharge (relative risk, 1.45; 95\% confidence interval, 0.41 to 5.16 ). The effect of ECPR on outcome in patients with OHCA may differ from those in patients with in-hospital cardiac arrest owing to many influencing factors that could be a reversible cause, such as no-flow time, low-flow time, witnessed arrest, and the quality of bystander CPR before ED arrival. Therefore, the selection of candidates for ECPR is important for successful implementation of ECPR in patients with OHCA. Further, ECPR is a resource intense- and timedependent procedure. The decision to perform ECPR is made discreetly in accordance with standardized protocols, not on a case-by-case basis according to the attending staff, to allow for rapid initiation. ${ }^{22}$

Most previous studies used the criteria of age $<75$ years and witnessed arrest for ECPR ${ }^{23-25}$ However, inclusion of other criteria such as CPR performed by bystander, initial shockable rhythm, and resuscitation duration is still being debated, and the literature suggests that there is an inverse relationship between CPR duration before ECPR and outcome. CPR provided for $<45 \mathrm{~min}-$ utes before ECPR has been associated with a survival-to-discharge rate of $57.1 \%$ compared with an $11.5 \%$ survival-to-discharge rate when the ECPR duration exceeds 60 minutes. ${ }^{26,27}$ In another study, a high mortality rate of 70\% was observed when CPR exceeded 60 minutes. In our study, we selected prehospital low-flow time $<30$ minutes as a criterion for eligibility because we aimed not to exceed 60 minutes of collapse-to-ECMO run time.
Recently, Grunau et al..$^{28}$ reported that approximately $10 \%$ of EMS-treated cases of OHCA fulfilled their hypothetical set of ECPR criteria. The variables of ECPR criteria suggested by Grunau et al. were similar to our criteria, except the age range; Grunau et al. included younger patients ( $<60$ years). According to them, their ECPR-eligible cohort demonstrated better outcomes with good neurologic outcomes of 35\% in contrast to $8 \%$ in our cohort. These differences might be attributable to the difference in the EMS system as well as the age of patients. There are regional differences in the EMS systems, and in Korea, EMS personnel rarely perform procedures such as epinephrine administration and advanced airway insertion. Thus, regional ECPR programs should be considered.

In our study, we found no difference in the prehospital variables between patients who received ECPR and those who received conventional CPR. However, the proportion of patients with good neurological outcomes at 1 month in the ECPR group (30.0\%) was more than 7-fold higher than that in the conventional CPR group (4.0\%). This result is consistent with the findings of previous studies and corroborates evidence on the potential benefit of ECPR in selective OHCA patients. ${ }^{7,810,11,13,21,22,29}$

Interestingly, the duration between ED arrival and implantation of ECMO was long; i.e., the mean time for patients with good neurological outcomes was 49 minutes, whereas the mean time for patients with poor neurological outcomes was 58 minutes. Despite the prolonged low-flow time in our study patients, the outcome was comparable to that of previous studies, which ranged between $10 \%$ and $30 \%$ in the rate of discharge with good neurological outcomes. ${ }^{7,8,10,11,13,21,22,29}$ These results indicate that for some OHCA patients who fulfill the ECPR criteria, termination of resuscitation efforts may not be allowed in a prehospital setting and these patients may have to be transferred to a facility where EMS personnel can attempt to resuscitate patients by performing ECPR. ${ }^{21}$

The main limitations of our present study are its retrospective design and the limited number of patients. First, because our criteria for ECPR eligibility were based on data from previous studies, characteristics not represented in our set of criteria may also be valuable for further identification of the ideal ECPR candidate. Second, during the study period, there were no ECPR eligibility criteria, and therefore, ECPR was performed in a case-by-case manner. Third, the generalizability of our results is limited because this study was conducted in a single tertiary medical center located in an urban area in Seoul, Korea. Finally, owing to the small number of patients who received ECPR $(n=10)$, the statistical power of our results was low.

In conclusion, approximately $11 \%$ of OHCA patients were eligible for ECPR in our study. Among those patients, only 4\% of 
patients (2/50) who did not receive ECPR survived with a good neurological outcome. Therefore, ECPR implementation might be a rescue option for increasing the probability of survival in potentially hopeless ECPR-eligible patients.

\section{CONFLICT OF INTEREST}

No potential conflict of interest relevant to this article was reported.

\section{REFERENCES}

1. Mozaffarian D, Benjamin EJ, Go AS, et al. Heart disease and stroke statistics: 2015 update. A report from the American Heart Association. Circulation 2015;131:e29-322.

2. Haneya A, Philipp A, Diez C, et al. A 5-year experience with cardiopulmonary resuscitation using extracorporeal life support in non-postcardiotomy patients with cardiac arrest. Resuscitation 2012;83:1331-7.

3. Chen YS, Lin JW, Yu HY, et al. Cardiopulmonary resuscitation with assisted extracorporeal life-support versus conventional cardiopulmonary resuscitation in adults with in-hospital cardiac arrest: an observational study and propensity analysis. Lancet 2008;372:554-61.

4. Kagawa $E_{1}$ Inoue I, Kawagoe $T$, et al. Assessment of outcomes and differences between in- and out-of-hospital cardiac arrest patients treated with cardiopulmonary resuscitation using extracorporeal life support. Resuscitation 2010;81:968-73.

5. Avalli L, Maggioni $E$, Formica $F$, et al. Favourable survival of in-hospital compared to out-of-hospital refractory cardiac arrest patients treated with extracorporeal membrane oxygenation: an Italian tertiary care centre experience. Resuscitation 2012;83:579-83.

6. Johnson NJ, Acker M, Hsu CH, et al. Extracorporeal life support as rescue strategy for out-of-hospital and emergency department cardiac arrest. Resuscitation 2014;85:1527-32.

7. Maekawa K, Tanno K, Hase M, Mori K, Asai Y. Extracorporeal cardiopulmonary resuscitation for patients with out-of-hospital cardiac arrest of cardiac origin: a propensity-matched study and predictor analysis. Crit Care Med 2013;41:1186-96.

8. Wang $\mathrm{CH}$, Chou NK, Becker LB, et al. Improved outcome of extracorporeal cardiopulmonary resuscitation for out-of-hospital cardiac arrest: a comparison with that for extracorporeal rescue for in-hospital cardiac arrest. Resuscitation 2014;85: 1219-24.

9. Dunne B, Christou E, Duff 0, Merry C. Extracorporeal-assisted rewarming in the management of accidental deep hypother- mic cardiac arrest: a systematic review of the literature. Heart Lung Circ 2014;23:1029-35.

10. Lamhaut $L$, Jouffroy $R$, Soldan $M$, et al. Safety and feasibility of prehospital extra corporeal life support implementation by non-surgeons for out-of-hospital refractory cardiac arrest. Resuscitation 2013;84:1525-9.

11. Sakamoto T, Morimura N, Nagao K, et al. Extracorporeal cardiopulmonary resuscitation versus conventional cardiopulmonary resuscitation in adults with out-of-hospital cardiac arrest: a prospective observational study. Resuscitation 2014; 85:762-8.

12. Shin TG, Choi JH, Jo IJ, et al. Extracorporeal cardiopulmonary resuscitation in patients with inhospital cardiac arrest: a comparison with conventional cardiopulmonary resuscitation. Crit Care Med 2011;39:1-7.

13. Stub D, Bernard S, Pellegrino V, et al. Refractory cardiac arrest treated with mechanical CPR, hypothermia, ECMO and early reperfusion (the CHEER trial). Resuscitation 2015;86:88-94.

14. Le Guen M, Nicolas-Robin A, Carreira S, et al. Extracorporeal life support following out-of-hospital refractory cardiac arrest. Crit Care 2011;15:R29.

15. Thiagarajan RR, Brogan TV, Scheurer MA, Laussen PC, Rycus PT, Bratton SL. Extracorporeal membrane oxygenation to support cardiopulmonary resuscitation in adults. Ann Thorac Surg 2009;87:778-85.

16. Jaski $B E$, Ortiz $B$, Alla $K R$, et al. A 20-year experience with urgent percutaneous cardiopulmonary bypass for salvage of potential survivors of refractory cardiovascular collapse. J Thorac Cardiovasc Surg 2010;139:753-7.e2.

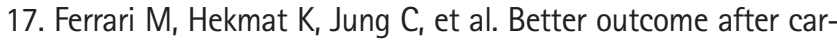
diopulmonary resuscitation using percutaneous emergency circulatory support in non-coronary patients compared to those with myocardial infarction. Acute Card Care 2011;13:30-4.

18. Cardarelli MG, Young AJ, Griffith B. Use of extracorporeal membrane oxygenation for adults in cardiac arrest (E-CPR): a meta-analysis of observational studies. ASAIO J 2009;55:581-6.

19. Tanno K, Itoh Y, Takeyama Y, Nara S, Mori K, Asai Y. Utstein style study of cardiopulmonary bypass after cardiac arrest. Am J Emerg Med 2008;26:649-54.

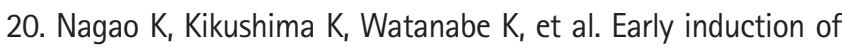
hypothermia during cardiac arrest improves neurological outcomes in patients with out-of-hospital cardiac arrest who undergo emergency cardiopulmonary bypass and percutaneous coronary intervention. Circ J 2010;74:77-85.

21. Ortega-Deballon I, Hornby L, Shemie SD, Bhanji F, Guadagno E. Extracorporeal resuscitation for refractory out-of-hospital cardiac arrest in adults: a systematic review of international 
practices and outcomes. Resuscitation 2016;101:12-20.

22. Mochizuki K, Imamura H, Iwashita T, Okamoto K. Neurological outcomes after extracorporeal cardiopulmonary resuscitation in patients with out-of-hospital cardiac arrest: a retrospective observational study in a rural tertiary care center. J Intensive Care 2014;2:33.

23. Conseil Francais de Reanimation Cardiopulmonaire; Societe Francaise d'Anesthesie et de Reanimation; Societe Francaise de Cardiologie, et al. Guidelines for indications for the use of extracorporeal life support in refractory cardiac arrest. French Ministry of Health. Ann Fr Anesth Reanim 2009;28:182-90.

24. Lazzeri $C$, Bernardo $P$, Sori $A$, et al. Venous-arterial extracorporeal membrane oxygenation for refractory cardiac arrest: a clinical challenge. Eur Heart J Acute Cardiovasc Care 2013;2: 118-26.

25. Poppe $M$, Weiser $C_{\text {, Holzer } M}$, et al. The incidence of "load\&go" out-of-hospital cardiac arrest candidates for emergency de- partment utilization of emergency extracorporeal life support: a one-year review. Resuscitation 2015;91:131-6.

26. Chen YS, Chao A, Yu HY, et al. Analysis and results of prolonged resuscitation in cardiac arrest patients rescued by extracorporeal membrane oxygenation. J Am Coll Cardiol 2003;41: 197-203.

27. Chen YS, Yu HY, Huang SC, et al. Extracorporeal membrane oxygenation support can extend the duration of cardiopulmonary resuscitation. Crit Care Med 2008;36:2529-35.

28. Grunau B, Scheuermeyer FX, Stub D, et al. Potential candidates for a structured Canadian ECPR program for out-of-hospital cardiac arrest. CJEM 2016:1-8.

29. Kim SJ, Jung JS, Park JH, Park JS, Hong YS, Lee SW. An optimal transition time to extracorporeal cardiopulmonary resuscitation for predicting good neurological outcome in patients with out-of-hospital cardiac arrest: a propensity-matched study. Crit Care 2014;18:535. 